\title{
Stimulators of soluble guanylate cyclase (sGC) inhibit experimental skin fibrosis of different aetiologies
}

\author{
Clara Dees, ${ }^{1}$ Christian Beyer, ${ }^{1}$ Alfiya Distler, ${ }^{1}$ Alina Soare, ${ }^{1,2,3}$ Yun Zhang, ${ }^{1}$ \\ Katrin Palumbo-Zerr, ${ }^{1}$ Oliver Distler, ${ }^{4}$ Georg Schett, ${ }^{1}$ Peter Sandner, ${ }_{1}^{5,6}$ \\ Jörg H W Distler ${ }^{1}$
}

Handling editor Tore K Kvien

- Additional material is published online only. To view please visit the journal online (http://dx.doi.org/10.1136/ annrheumdis-2014-206809).

For numbered affiliations see end of article.

\section{Correspondence to}

Professor Jörg H W Distler, Department of Medicine 3 , Institute for Clinical Immunology, University of Erlangen-Nuremberg, Ulmenweg 18, Erlangen D-91054, Germany; Joerg.distler@uk-erlangen.de

Received 16 October 2014 Revised 13 February 2015

Accepted 7 March 2015 Published Online First 27 March 2015

\section{CrossMark}

To cite: Dees C, Beyer C, Distler $A$, et al. Ann Rheum Dis 2015:74:1621-1625.

\section{ABSTRACT}

Objectives Stimulators of the soluble guanylate cyclase $(\mathrm{sGC})$ have recently been shown to inhibit transforming growth factor- $\beta$ signalling. Here, we aimed to demonstrate that riociguat, the drug candidate for clinical trials in systemic sclerosis (SSc), is effective in experimental fibrosis and to compare its efficacy to that of phosphodiesterase $V$ inhibitors that also increase the intracellular levels of cyclic guanosine monophosphate.

Methods The antifibrotic effects of riociguat and sildenafil were compared in the tight-skin 1 model, in bleomycin-induced fibrosis and in a model of sclerodermatous chronic graft-versus-host-disease (cGvHD). Doses of $0.1-3 \mathrm{mg} / \mathrm{kg}$ twice a day for riociguat and of 3-10 mg/kg twice a day for sildenafil were used.

Result Riociguat dose-dependently reduced skin thickening, myofibroblast differentiation and accumulation of collagen with potent antifibrotic effects at 1 and $3 \mathrm{mg} / \mathrm{kg}$. Riociguat also ameliorated fibrosis of the gastrointestinal tract in the cGvHD model. The antifibrotic effects were associated with reduced phosphorylation of extracellular signal-regulated kinases. Sildenafil at doses of 3 and $10 \mathrm{mg} / \mathrm{kg}$ exerted mild antifibrotic effects that were significantly less pronounced compared with 1 and $3 \mathrm{mg} / \mathrm{kg}$ riociguat.

Conclusions These data demonstrated potent antifibrotic effects of riociguat on experimental skin and organ fibrosis. These findings suggest a role for riociguat for the treatment of fibrotic diseases, especially for the treatment of SSC. A phase II study with riociguat in patients with SSc is currently starting.

Systemic sclerosis (SSc) is characterised by fibrosis and vascular alterations, both of which account for the high morbidity and mortality of SSc. ${ }^{1}$ Although several therapies are in clinical use for the treatment of vascular manifestations, no targeted therapies are yet approved for the treatment of fibrosis. Thus, there is a great medical need for novel therapies.

Recent studies indicated that the soluble guanylate cyclase (sGC), which catalyses the production of cyclic guanosine monophosphate (cGMP) from guanosine triphosphate, ${ }^{2}{ }^{3}$ might be an interesting molecular target for the treatment of vascular manifestations and tissue fibrosis in SSc. ${ }^{4}$ Riociguat, a stimulator of sGC, has recently been approved as a novel, first-in-class drug for the treatment of pulmonary arterial hypertension (PAH) after showing efficacy and good tolerability in the PATENT studies involving $>400$ patients with idiopathic pulmonary arterial hypertension and connective tissue disease-PAH. ${ }^{6}$ We demonstrated that the $\mathrm{sGC}$ stimulator, BAY-41-2272, which served as an early lead compound for pharmaceutical development, ${ }^{7}$ inhibits transforming growth factor- $\beta$ (TGF- $\beta$ )induced fibroblast activation and ameliorates experimental skin fibrosis. ${ }^{5}$ Here, we aimed to provide additional experimental data to support the potential of sGC stimulators in SSc. We address two limitations of our previous studies and demonstrate that (1) not only BAY-41-2272 but also riociguat exerts antifibrotic effects in preclinical models of SSc at well-tolerated doses and that (2) sGC stimulators not only inhibit experimental skin fibrosis but are also effective in models of systemic fibrosis. Finally, we aim to directly compare the antifibrotic effects of sGC stimulators with those of phosphodiesterase V (PDE5) inhibitor sildenafil. As for sGC stimulators, PDE5 inhibitors such as sildenafil also increase the intracellular levels of cGMP; however, in contrast to sGC stimulators, they do not stimulate the production of cGMP, but inhibit its degradation. ${ }^{3}$ These experiments will provide first evidence whether the deficiency in cGMP in SSc fibroblasts is primarily caused by impaired formation or enhanced degradation of cGMP.

\section{MATERIALS AND METHODS \\ Mouse models}

Tsk-1 mice

Tight-skin 1 (Tsk-1) mice are characterised by endogenous, TGF- $\beta$-dependent activation of fibroblasts. ${ }^{8}$ Treatment of Tsk-1 mice with riociguat as well as treatment of control mice started at the age of 5 weeks and was continued for 5 weeks. ${ }^{9} 10$ Littermates not carrying the Tsk-1 allele and vehicle-treated Tsk-1 mice served as controls.

\section{Bleomycin-induced dermal fibrosis}

Skin fibrosis was induced in $\mathrm{DBA} / 2$ mice by bleomycin. ${ }^{811}$ The outcome was analysed after 3 weeks of treatment with riociguat and sildenafil. Mice injected with $0.9 \%$ sodium chloride served as controls.

\section{Sclerodermatous chronic graft-versus-host disease (cGvHD)}

The B10.D2 $\rightarrow$ Balb/c (H-2(d)) minor histocompatibility antigen-mismatched model was used as a model of SSc with systemic fibrosis. ${ }^{8} 1213$ cGvHD was induced by allogeneic transplantation of $5 \times 10^{6}$ splenocytes and $1 \times 10^{6}$ bone marrow cells 
from B10.D2 mice into BALB/c (H-2d) mice as described. $\mathrm{BALB} / \mathrm{c}(\mathrm{H}-2 \mathrm{~d})$ mice transplanted with splenocytes and bone marrow cells isolated from $\mathrm{BALB} / \mathrm{c}(\mathrm{H}-2 \mathrm{~d})$ mice served as controls. Treatment started 10 days after transplantation, and the outcome was analysed after 6 weeks.

\section{Pharmacological treatment}

Riociguat and sildenafil (both kindly provided by Bayer Pharma, Wuppertal, Germany) were dissolved in $0.5 \%$ methylcellulose and applied by oral gavage. Mice were treated with riociguat in doses of $0.1,0.3,1$ and $3 \mathrm{mg} / \mathrm{kg}$ twice a day. Sildenafil was given in doses of 3 and $10 \mathrm{mg} / \mathrm{kg}$ twice a day. Mice treated with $0.5 \%$ methylcellulose served as controls. Methotrexate (MTX) was used as reference and injected intraperitoneally at a dose of $2.5 \mathrm{mg} / \mathrm{kg}$ once daily as used previously. ${ }^{14}$

\section{Evaluation}

Skin fibrosis was assessed by analysis of dermal thickening, evaluation of the hydroxyproline content and quantification of the number of $\alpha$-smooth muscle actin-positive myofibroblasts. ${ }^{15-17}$ Extracellular signal-regulated kinases (ERK)1/2 and pERK1/2 were analysed by immunohistochemical staining.
Fibrosis of the duodenum was quantified by Pico Sirius red staining and by analyses of the hydroxyproline content.

\section{Statistical analysis}

Data are expressed as median \pm IQR and differences between the groups were tested for their statistical significance by nonparametric Mann-Whitney U test. p Values $<0.05$ were considered significant.

\section{RESULTS}

\section{Riociguat ameliorates fibrosis in Tsk-1 mice}

Treatment with riociguat exerted dose-dependent antifibrotic effects in the Tsk-1 model (figure 1A-D). Whereas only trends but no significant antifibrotic effects were observed at doses of $0.1 \mathrm{mg} / \mathrm{kg}$, moderate but significant reductions in hypodermal thickening, hydroxyproline content and myofibroblast counts were observed at doses of $0.3 \mathrm{mg} / \mathrm{kg}$. Potent and comparable antifibrotic effects were observed with 1 and $3 \mathrm{mg} / \mathrm{kg}$ riociguat with reduction in hypodermal thickening of the hydroxyproline content and of myofibroblast counts compared with vehicletreated Tsk-1 mice. Treatment with MTX or sildenafil $(10 \mathrm{mg} / \mathrm{kg})$ also reduced fibrosis, but the antifibrotic effects were
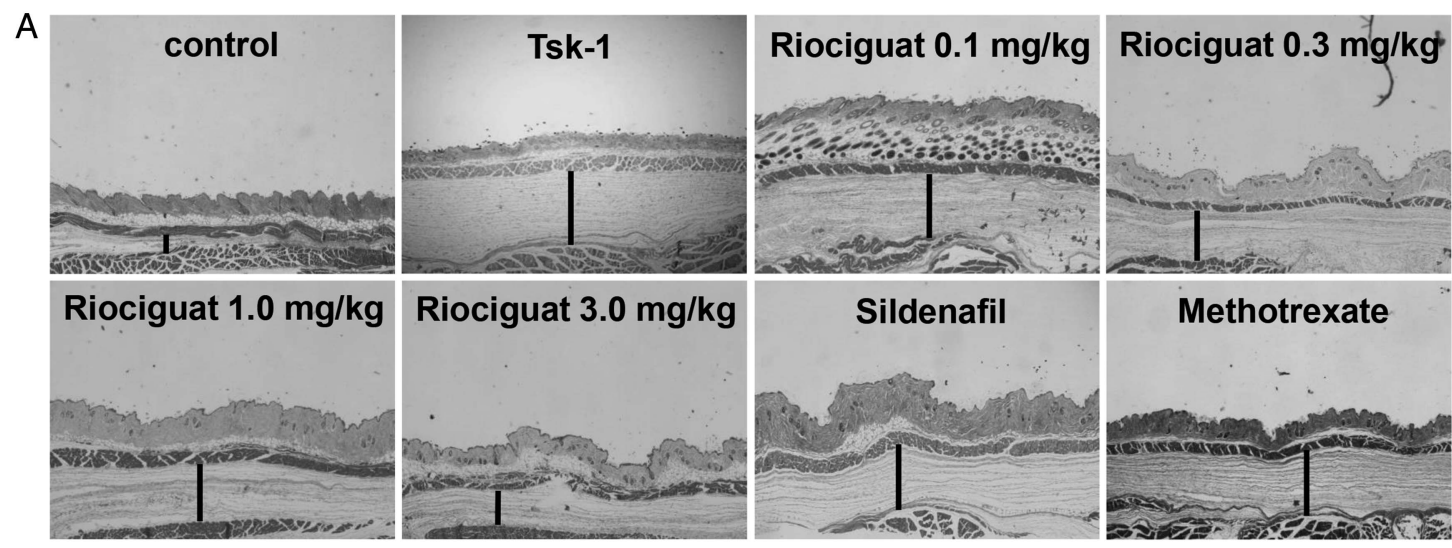

Methotrexate

B

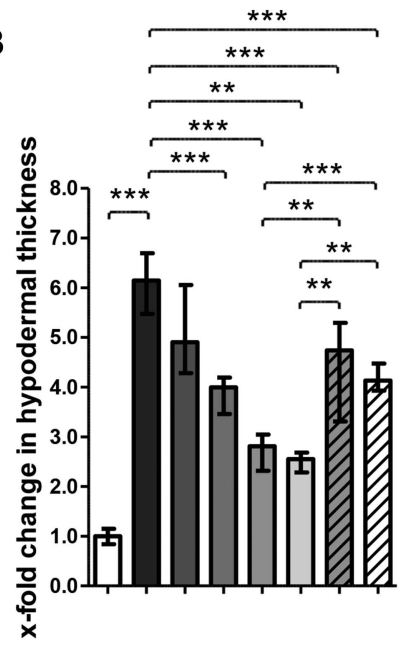

Tsk-1 + Riociguat $0.1 \mathrm{mg} / \mathrm{kg}$

Tsk-1 + Riociguat $0.3 \mathrm{mg} / \mathrm{kg}$

Tsk-1 + Riociguat $1 \mathrm{mg} / \mathrm{kg}$
Tsk-1 + Riociguat $3 \mathrm{mg} / \mathrm{kg}$

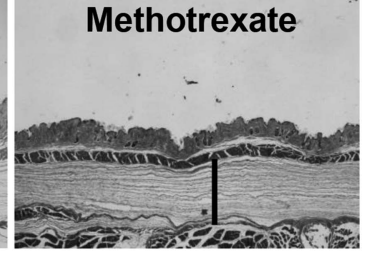

C
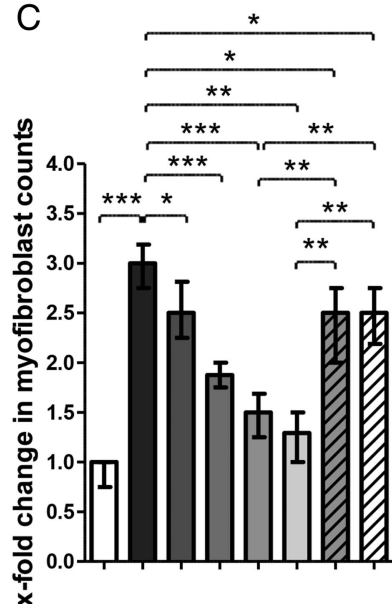

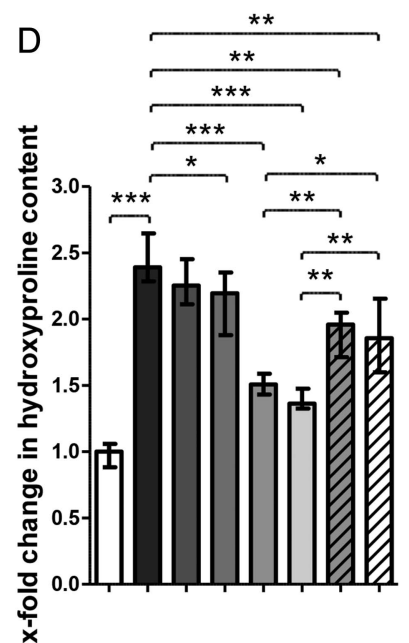

Figure 1 Treatment with riociguat ameliorates fibrosis in the tight-skin 1 (Tsk-1) model. (A) Representative images of HE-stained skin sections for each group of mice are shown at 40-fold magnification. Vertical bars indicate hypodermal thickness. Quantification of fibrosis by assessment of the hypodermal thickness (B), the number of $\alpha$-smooth muscle actin-positive myofibroblasts (C) and the hydroxyproline content (D). All groups consisted of eight mice each. 
only mild and within the range of that observed with low doses of $0.1-0.3 \mathrm{mg} / \mathrm{kg}$ riociguat (figure $1 \mathrm{~A}-\mathrm{D}$ ).

\section{Riociguat is more effective than sildenafil in bleomycin-induced skin fibrosis}

We next evaluated riociguat in bleomycin-induced fibrosis and compared its antifibrotic effects with that of the PDE5 inhibitor sildenafil. Treatment with riociguat exerted dose-dependent antifibrotic effects in concentrations between 0.3 and $3 \mathrm{mg} / \mathrm{kg}$ and strongly reduced dermal thickening, hydroxyproline content and myofibroblast differentiation in higher concentrations without evidence of adverse effects (figure 2A-D). In contrast to stimulation of cGMP production by riociguat, inhibition of its degradation by sildenafil was less effective. Even in high doses of $10 \mathrm{mg} / \mathrm{kg}$ sildenafil showed only mild antifibrotic effects in bleomycin-induced fibrosis (figure 2A-D).

\section{Riociguat inhibits systemic fibrosis in experimental cGvHD}

To demonstrate the effects of riociguat on other fibrotic manifestations, we tested its efficacy in a model of sclerodermatous cGvHD with multiorgan involvement. Treatment with riociguat did not only strongly ameliorate cGvHD-induced skin fibrosis (figure 3A-D) but also inhibited fibrosis of the intestinal tract (figure $3 \mathrm{E}, \mathrm{F}$ ). Sirius red staining and hydroxyproline content in the small intestine were significantly reduced upon treatment with riociguat compared with vehicle treatment (figure $3 \mathrm{~B}$ ). Consistent with the findings in bleomycin-induced skin fibrosis, sildenafil was less effective and demonstrated only mild antifibrotic effects on cGvHD-induced fibrosis of the skin and the small intestine (figure 3).

In all three preclinical models, we did not observe any differences in body weight, food consumption, consistency of the stool, texture of the skin or activity for any group of mice treated with riociguat. We also did not observe pathological changes on necropsy.

Consistent with our previous in vitro data, ${ }^{5}$ riociguat inhibited non-canonical, Erk-dependent TGF- $\beta$ signalling and reduced the levels of phosphorylated ERK1/2 in all three mouse models. In contrast, the effects of sildenafil on phosphorylated ERK1/2 were less pronounced (see online supplementary figures S1-S3).

\section{DISCUSSION}

We demonstrate in the present study that riociguat dosedependently inhibits fibrosis in models of early inflammatory stages of SSc such as the mouse model of bleomycin-induced skin fibrosis and in models of later, less-inflammatory stages of SSc with endogenous activation of fibroblasts such as the Tsk-1 model. Potent antifibrotic effects were observed in pharmacologically relevant doses of 1 and $3 \mathrm{mg} / \mathrm{kg}$. Consistent with the

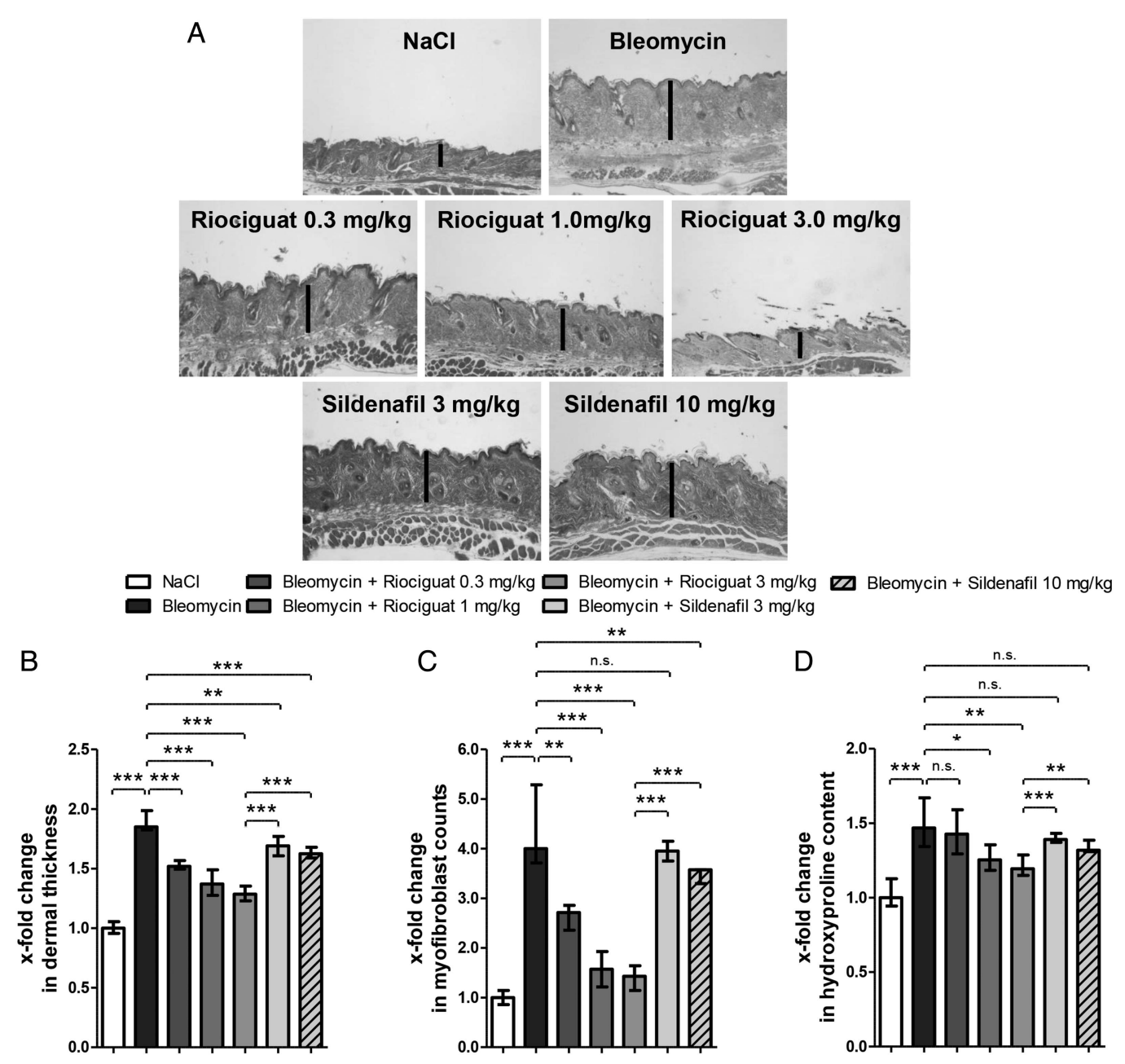

Figure 2 Riociguat is more effective than sildenafil in bleomycin-induced skin fibrosis. (A) Representative images of HE-stained skin sections are shown at 100-fold magnification. Vertical bars indicate dermal thickness. Effects of riociguat and sildenafil on the dermal thickness (B), myofibroblast counts (C) and hydroxyproline content (D). All groups consisted of eight mice each. 
A

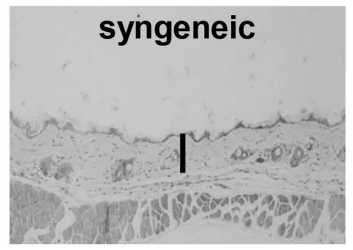

allogeneic

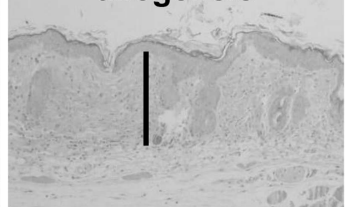

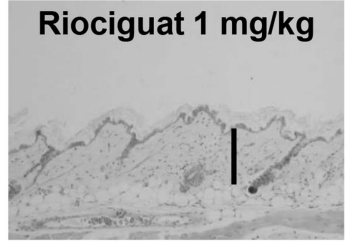

Sildenafil $10 \mathrm{mg} / \mathrm{kg}$

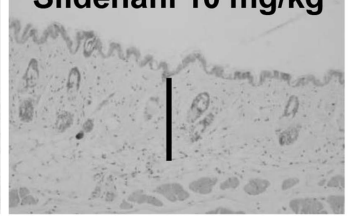

$\mathrm{D}$

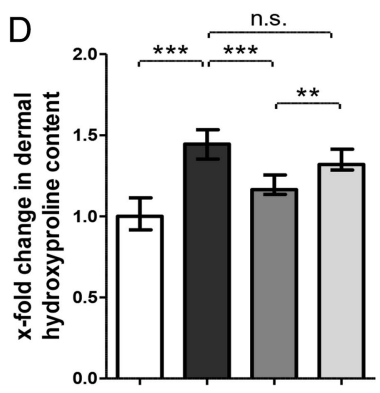

$\mathrm{E}$

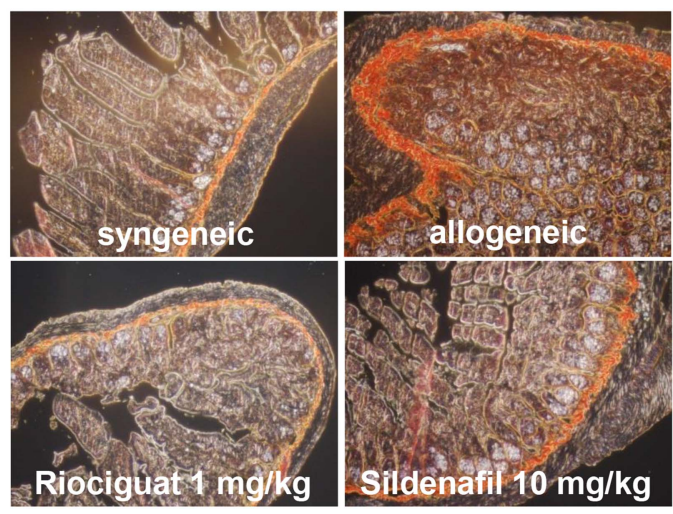

C

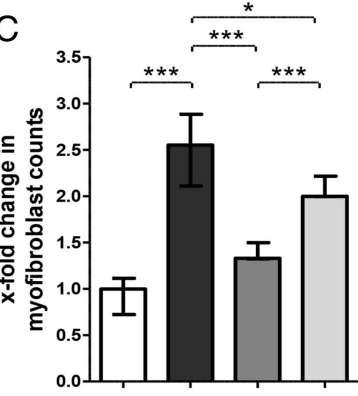

$\mathrm{F}$

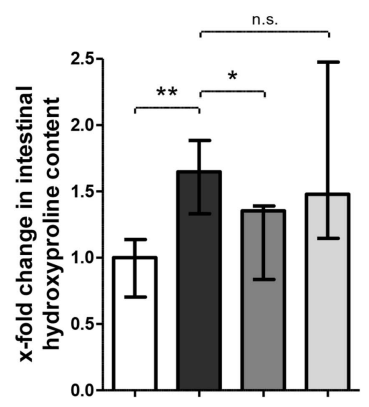

Figure 3 Riociguat reduces skin and intestinal fibrosis in experimental sclerodermatous chronic graft-versus-host-disease (cGvHD). (A-D) Effects of riociguat and sildenafil on CGvHD-induced skin fibrosis: (A) representative images of HE-stained sections at 100-fold magnification, (B) quantification of the dermal thickness, (C) myofibroblast counts and (D) hydroxyproline content. Vertical bars in HE-stained sections indicate dermal thickness. $(E$ and $F)$ Effects of riociguat and sildenafil on fibrosis of the small intestine: $(E)$ representative images of Sirius red-stained samples at 200-fold magnification and (F) hydroxyproline content. All groups consisted of eight mice each.

inhibitory effects of sGC stimulators on TGF- $\beta$ signalling as a common core pathway of fibrosis, riociguat was not only effective in experimental skin fibrosis but also in a model of sclerodermatous cGvHD with gastrointestinal involvement and has been reported to be effective in bleomycin-induced pulmonary fibrosis. Thus, there is strong evidence for antifibrotic effects of sGC stimulators in preclinical models resembling different stages and organ manifestations of SSc. These findings suggest a role for riociguat for the treatment of SSc. Moreover, sGC stimulation has also been shown to ameliorate fibrosis in other organs commonly affected in SSc such as the lungs and the heart. ${ }^{18} 19$ These findings suggest a role for riociguat for the treatment of fibrotic diseases, especially for the treatment of SSc-driven internal organ involvement. Currently, a phase II study with riociguat in patients with SSc is ongoing. sGC stimulators might not only inhibit fibrosis but may also improve vascular manifestations of SSc such as PAH. sGC stimulators may also ameliorate Raynaud's attacks and improve ulcer healing in SSc. $^{712}$ Further studies are required to assess the effects of sGC stimulators on microvascular disease in SSc. However, its vasodilative effects were also underlying the most common adverse events of riociguat in PAH trials such as hypotension, headache and dizziness. ${ }^{6}$ Other adverse events such as dyspepsia and anaemia were rare, and riociguat was well-tolerated in patients with $\mathrm{PAH}$.
sGC stimulators exert their intracellular effects by increasing the intracellular levels of cGMP. ${ }^{7}$ Encouraged by the efficacy of sGC stimulators in experimental fibrosis, we tested the antifibrotic effects of PDE5 inhibition as an alternative pharmacological approach to increase the intracellular levels of cGMP. In contrast to sGC stimulators, PDE5 inhibitors do not enhance the formation of cGMP, but inhibit one of the major cGMP hydrolysing enzymes. ${ }^{3}$ Consistent with recent clinical findings in idiopathic pulmonary fibrosis, ${ }^{20}$ treatment with sildenafil demonstrated small but significant antifibrotic effects in experimental models of SSc. However, PDE5 inhibition was less effective than sGC stimulation with constantly less potent antifibrotic effects in all readouts. Therefore, sGC stimulators as riociguat may provide an antifibrotic treatment option for patients with SSc targeting both skin fibrosis and fibrosis of internal organs.

\section{Author affiliations}

${ }^{1}$ Department of Internal Medicine 3,Institute for Clinical Immunology, University of Erlangen-Nuremberg, Germany

${ }^{2}$ Internal Medicine and Rheumatology Department, Dr. I. Cantacuzino Clinical Hospital, Bucharest, Romania

${ }^{3}$ Carol Davila University of Medicine and Pharmacy, Bucharest, Romania

${ }^{4}$ Center of Experimental Rheumatology, Research of Systemic Autoimmune Diseases, University Hospital Zurich, Switzerland

${ }^{5}$ Bayer Health Care, Global Drug Discovery—Common Mechanism Research, Wuppertal, Germany

${ }^{6}$ Hannover Medical School, Institute of Pharmacology, Hannover, Germany 
Acknowledgements We thank Katja Dreißigacker, Regina Kleinlein and Christiane Bliß for excellent technical assistance.

Contributors Design of the study: $C D, C B, P S$ and JHWD. Acquisition of data: $C D$, $C B, A D, A S, Y Z, A S$ and $K P-Z$. Interpretation of data: $C D, C B, A D, O D, G S, P S$ and JHWD. Manuscript preparation: CD, PS and JHWD.

Funding Grants DI 1537/4-1, DI 1537/5-1, DI 1537/7-1, DI 1537/8-1, DI 1537/9-1, BE 5191/1-1, AK 144/1-1 and SCHE 1583/7-1 of the Deutsche Forschungsgesellschaft, grants A57, J29 and J39 of the IZKF Erlangen, the ELAN-Program of the University of Erlangen-Nuremberg and the Career Support Award of Medicine of the Ernst Jung Foundation. AS received a travel bursary from the European League against Rheumatism (EULAR).

Competing interests OD has consultancy relationships and/or has received research funding from Actelion, Pfizer, Ergonex, BMS, Sanofi-Aventis, United BioSource Corporation, Medac, Biovitrium, Boehringer Ingelheim, Bayer Pharma AG, Novartis, 4D Science and Active Biotech in the area of potential treatments of SSC. JHWD has consultancy relationships and/or has received research funding from Actelion, Active Biotech, Array Biopharma, Bayer Pharma AG, Boehringer Ingelheim, Celgene, GlaxoSmithKline, JB Therapeutics, Karo Bio, Novartis, Sanofi-Aventis, SigmaTau, UCB Pharma and in the area of potential treatments of SSC and is stock owner of $4 \mathrm{D}$ Science GmbH. PS is an employee of Bayer Pharma AG.

Provenance and peer review Not commissioned; externally peer reviewed.

\section{REFERENCES}

1 Gabrielli A, Avvedimento EV, Krieg T. Scleroderma. N Engl J Med 2009;360:1989-2003.

2 Derbyshire ER, Marletta MA. Structure and regulation of soluble guanylate cyclase. Annu Rev Biochem 2012;81:533-59.

3 Evora PR, Evora PM, Celotto AC, et al. Cardiovascular therapeutics targets on the NO-sGC-cGMP signaling pathway: a critical overview. Curr Drug Targets 2012;13:1207-14.

4 Beyer C, Reich N, Schindler SC, et al. Stimulation of soluble guanylate cyclase reduces experimental dermal fibrosis. Ann Rheum Dis 2012;71:1019-26.

5 Beyer C, Zenzmaier C, Palumbo-Zerr K, et al. Stimulation of the soluble guanylate cyclase (sGC) inhibits fibrosis by blocking non-canonical TGFbeta signalling. Ann Rheum Dis 2015;74:1408-16.

6 Ghofrani HA, Galie N, Grimminger F, et al. Riociguat for the treatment of pulmonary arterial hypertension. N Engl J Med 2013;369:330-40.
7 Boerrigter G, Burnett JC Jr. Nitric oxide-independent stimulation of soluble guanylate cyclase with BAY 41-2272 in cardiovascular disease. Cardiovasc Drug Rev 2007; 25:30-45.

8 Beyer C, Schett G, Distler O, et al. Animal models of systemic sclerosis: prospects and limitations. Arthritis Rheum 2010;62:2831-44.

9 Akhmetshina A, Palumbo K, Dees C, et al. Activation of canonical Wnt signalling is required for TGF-beta-mediated fibrosis. Nat Commun 2012;3:735.

10 Reich $\mathrm{N}$, Tomcik $\mathrm{M}$, Zerr $\mathrm{P}$, et al. Jun $\mathrm{N}$-terminal kinase as a potential molecular target for prevention and treatment of dermal fibrosis. Ann Rheum Dis 2012;71:737-45

11 Dees C, Akhmetshina A, Zerr P, et al. Platelet-derived serotonin links vascular disease and tissue fibrosis. J Exp Med 2011;208:961-72.

12 Zerr P, Palumbo-Zerr K, Distler A, et al. Inhibition of hedgehog signaling for the treatment of murine sclerodermatous chronic graft-versus-host disease. Blood 2012;120:2909-17.

13 Zerr P, Distler A, Palumbo-Zerr K, et al. Combined inhibition of c-Abl and PDGF receptors for prevention and treatment of murine sclerodermatous chronic graft-versus-host disease. Am J Pathol 2012;181:1672-80.

14 Lange F, Baitner E, Rintisch C, et al. Methotrexate ameliorates $T$ cell dependent autoimmune arthritis and encephalomyelitis but not antibody induced or fibroblast induced arthritis. Ann Rheum Dis 2005;64:599-605.

15 Dees C, Tomcik M, Palumbo-Zerr K, et al. JAK-2 as a novel mediator of the profibrotic effects of transforming growth factor beta in systemic sclerosis. Arthritis Rheum 2012;64:3006-15.

16 Dees $C$, Tomcik M, Zerr $P$, et al. Notch signalling regulates fibroblast activation and collagen release in systemic sclerosis. Ann Rheum Dis 2011;70:1304-10.

17 Horn A, Kireva T, Palumbo-Zerr K, et al. Inhibition of hedgehog signalling prevents experimental fibrosis and induces regression of established fibrosis. Ann Rheum Dis 2012;71:785-9.

18 Masuyama H, Tsuruda T, Sekita Y, et al. Pressure-independent effects of pharmacological stimulation of soluble guanylate cyclase on fibrosis in pressure-overloaded rat heart. Hypertens Res 2009;32:597-603.

19 Egemnazarov B, Sydykov A, Schermuly RT, et al. Novel soluble guanylyl cyclase stimulator BAY 41-2272 attenuates ischemia-reperfusion-induced lung injury. Am J Physiol Lung Cell Mol Physiol 2009;296:L462-469.

20 Zisman DA, Schwarz M, Anstrom KJ, et al. A controlled trial of sildenafil in advanced idiopathic pulmonary fibrosis. $N$ Engl I Med 2010;363:620-8. 


\section{ARD Stimulators of soluble guanylate cyclase (sGC) inhibit experimental skin fibrosis of different aetiologies}

Clara Dees, Christian Beyer, Alfiya Distler, Alina Soare, Yun Zhang, Katrin Palumbo-Zerr, Oliver Distler, Georg Schett, Peter Sandner and Jörg H W Distler

Ann Rheum Dis 2015 74: 1621-1625 originally published online March 27, 2015

doi: 10.1136/annrheumdis-2014-206809

Updated information and services can be found at:

http://ard.bmj.com/content/74/8/1621

These include:

Supplementary Material

References

Email alerting service
Supplementary material can be found at:

http://ard.bmj.com/content/suppl/2015/03/27/annrheumdis-2014-2068 09.DC1

This article cites 20 articles, 9 of which you can access for free at: http://ard.bmj.com/content/74/8/1621\#BIBL

Receive free email alerts when new articles cite this article. Sign up in the box at the top right corner of the online article.

Topic Articles on similar topics can be found in the following collections

Collections Connective tissue disease (4253)

\section{Notes}

To request permissions go to:

http://group.bmj.com/group/rights-licensing/permissions

To order reprints go to:

http://journals.bmj.com/cgi/reprintform

To subscribe to BMJ go to:

http://group.bmj.com/subscribe/ 